\title{
Supplementary material - Variability of mass changes at basin scale for Greenland and Antarctica
}

V. R. Barletta ${ }^{1}$, L. S. Sørensen ${ }^{1}$, and R. Forsberg ${ }^{1}$

${ }^{1}$ Geodynamics Department, DTU Space, Elektrovej 2800 Kgs. Lyngby, Denmark 

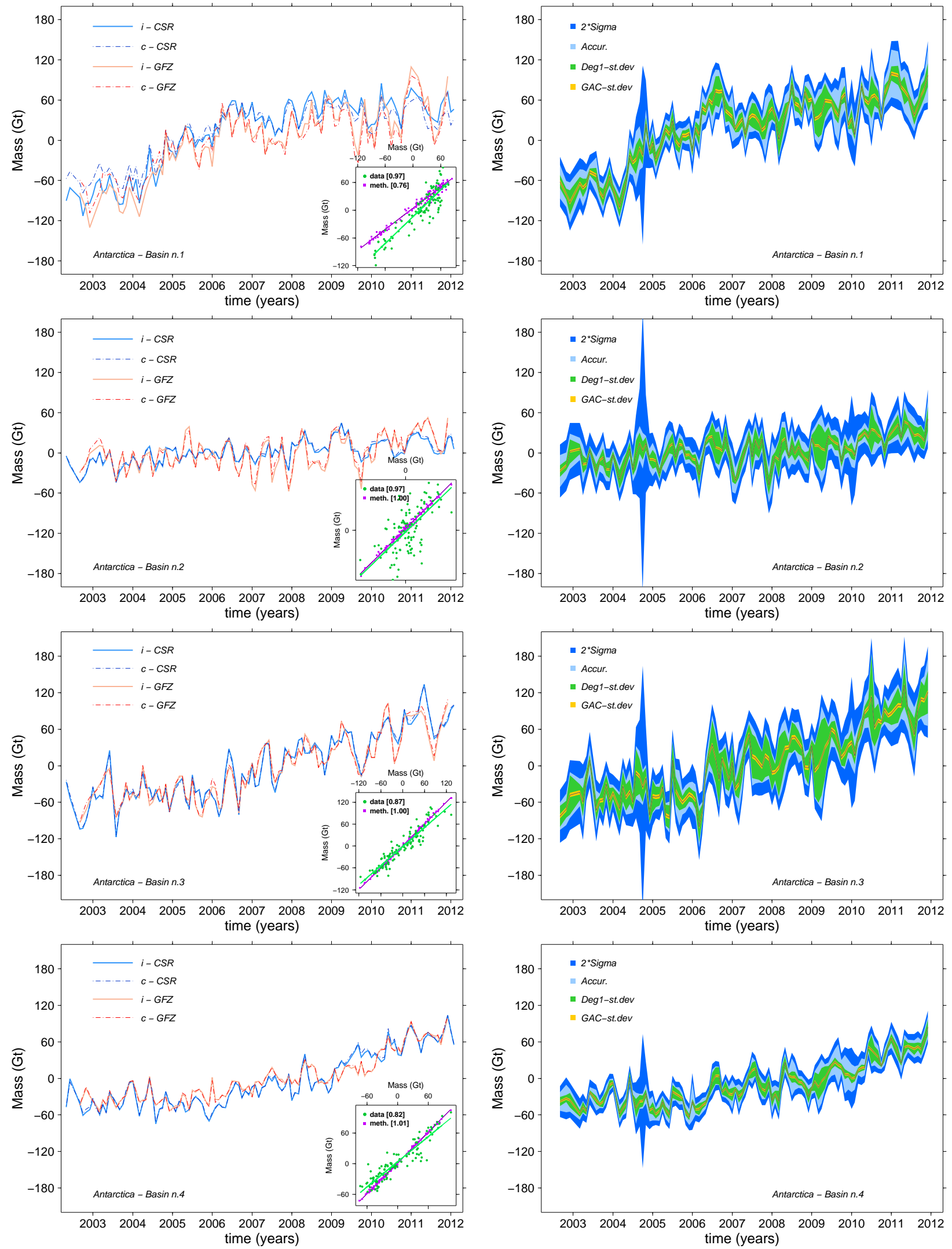

Fig. SM. 1. RL04 monthly solution for Antarctica. Basin number is indicated in each plot. Left column represents the comparison between the two methods and two data sets (CSR and GFZ) as in Fig.3 in the main text. Right column represents the monthly average solution where each color in the band around the average represents a contribution to the error estimate as in Fig. 7 of the main text. 

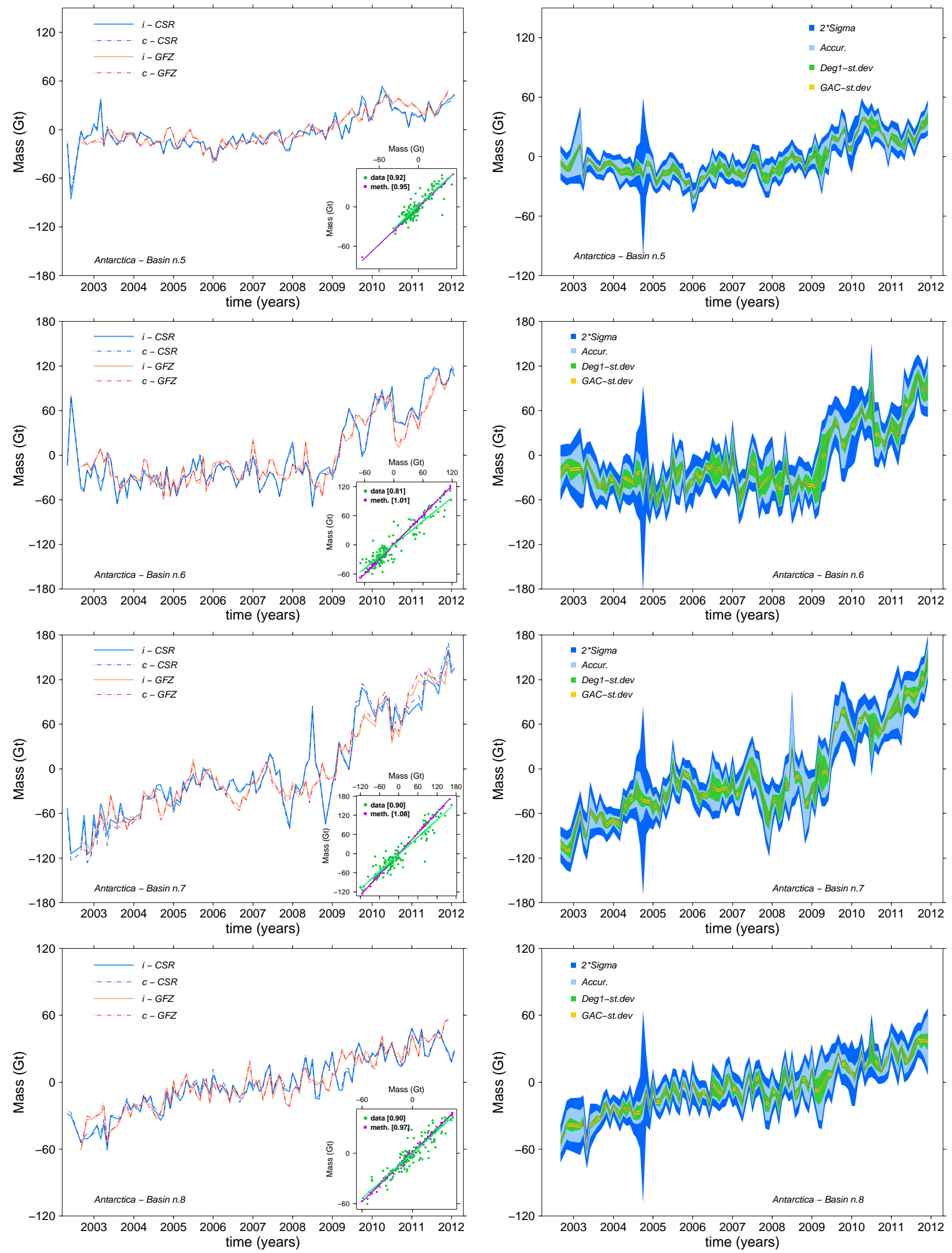

Fig. SM. 2. RL04 monthly solution for Antarctica. Basin number is indicated in each plot. Left column represents the comparison between the two methods and two data sets (CSR and GFZ) as in Fig.3 in the main text. Right column represents the monthly average solution where each color in the band around the average represents a contribution to the error estimate as in Fig. 7 of the main text. 

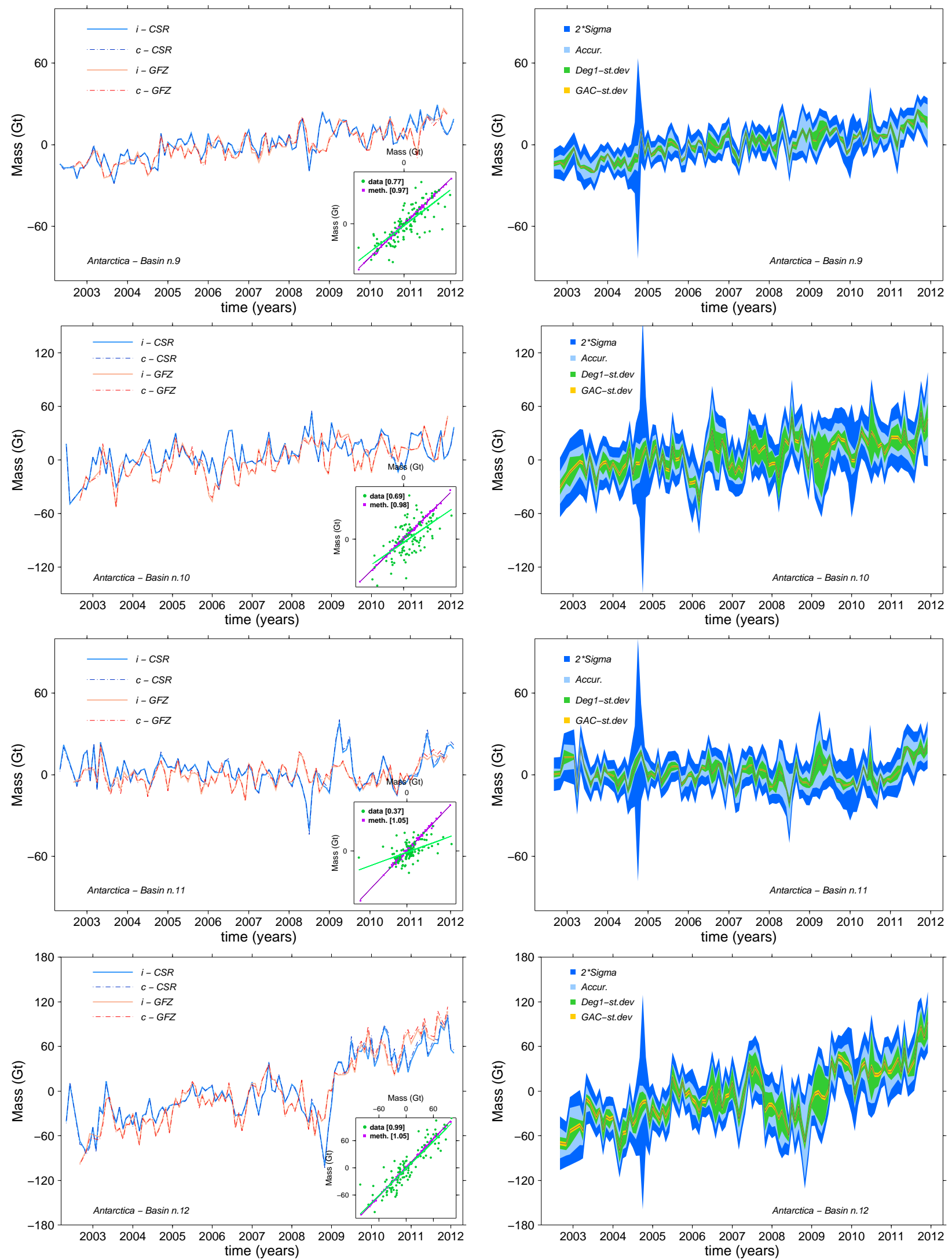

Fig. SM. 3. RL04 monthly solution for Antarctica. Basin number is indicated in each plot. Left column represents the comparison between the two methods and two data sets (CSR and GFZ) as in Fig.3 in the main text. Right column represents the monthly average solution where each color in the band around the average represents a contribution to the error estimate as in Fig. 7 of the main text. 

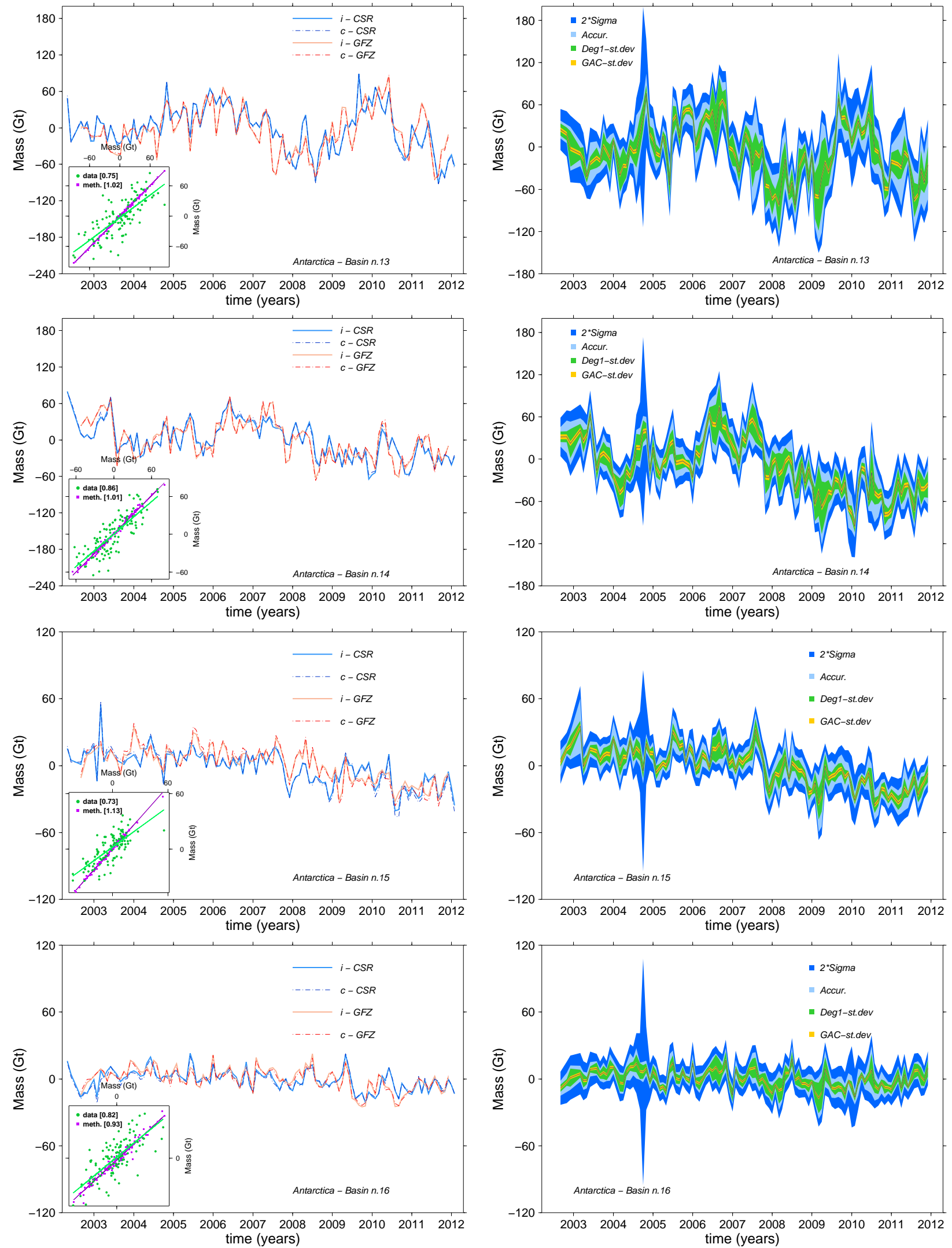

Fig. SM. 4. RL04 monthly solution for Antarctica. Basin number is indicated in each plot. Left column represents the comparison between the two methods and two data sets (CSR and GFZ) as in Fig.3 in the main text. Right column represents the monthly average solution where each color in the band around the average represents a contribution to the error estimate as in Fig. 7 of the main text. 

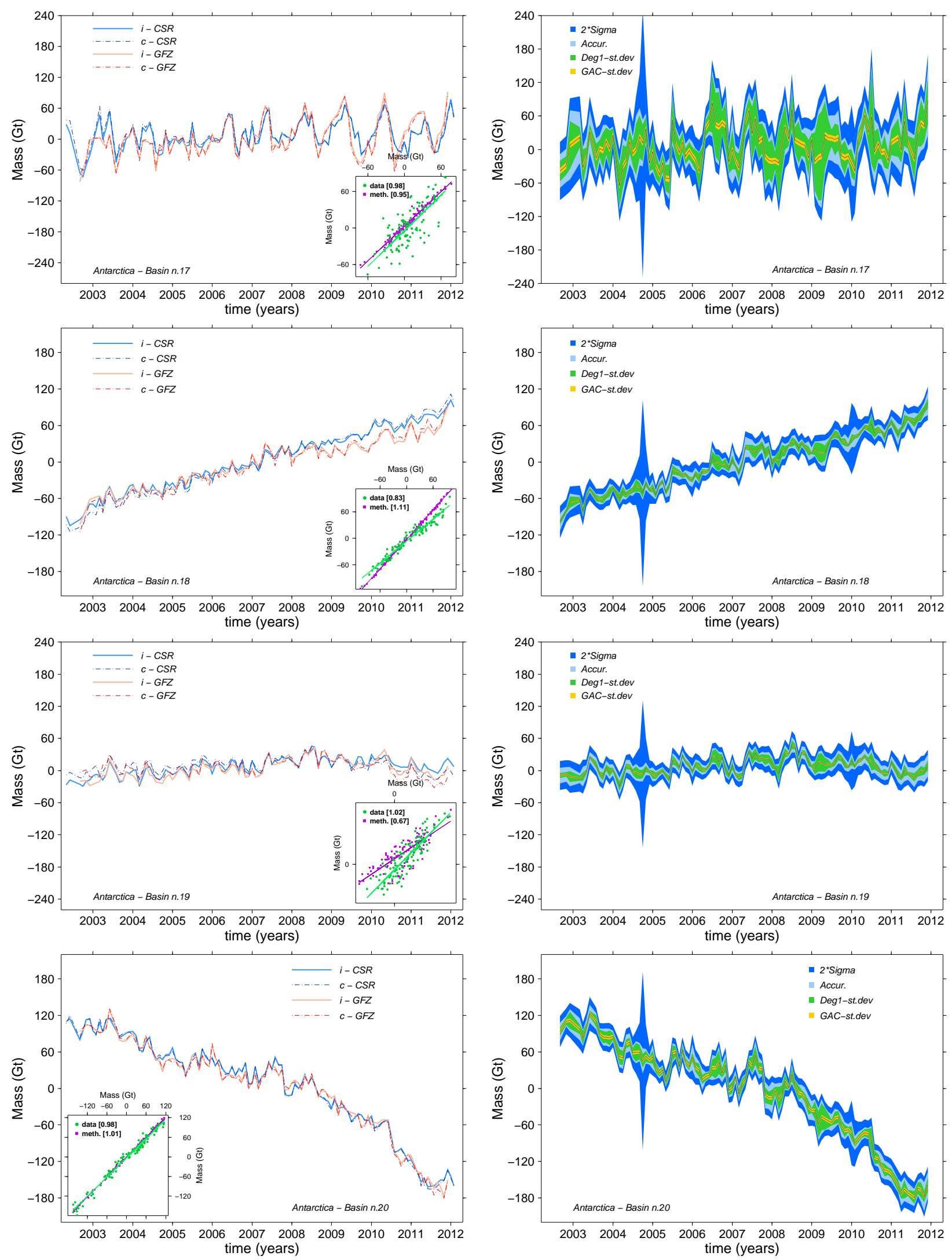

Fig. SM. 5. RL04 monthly solution for Antarctica. Basin number is indicated in each plot. Left column represents the comparison between the two methods and two data sets (CSR and GFZ) as in Fig.3 in the main text. Right column represents the monthly average solution where each color in the band around the average represents a contribution to the error estimate as in Fig. 7 of the main text. 

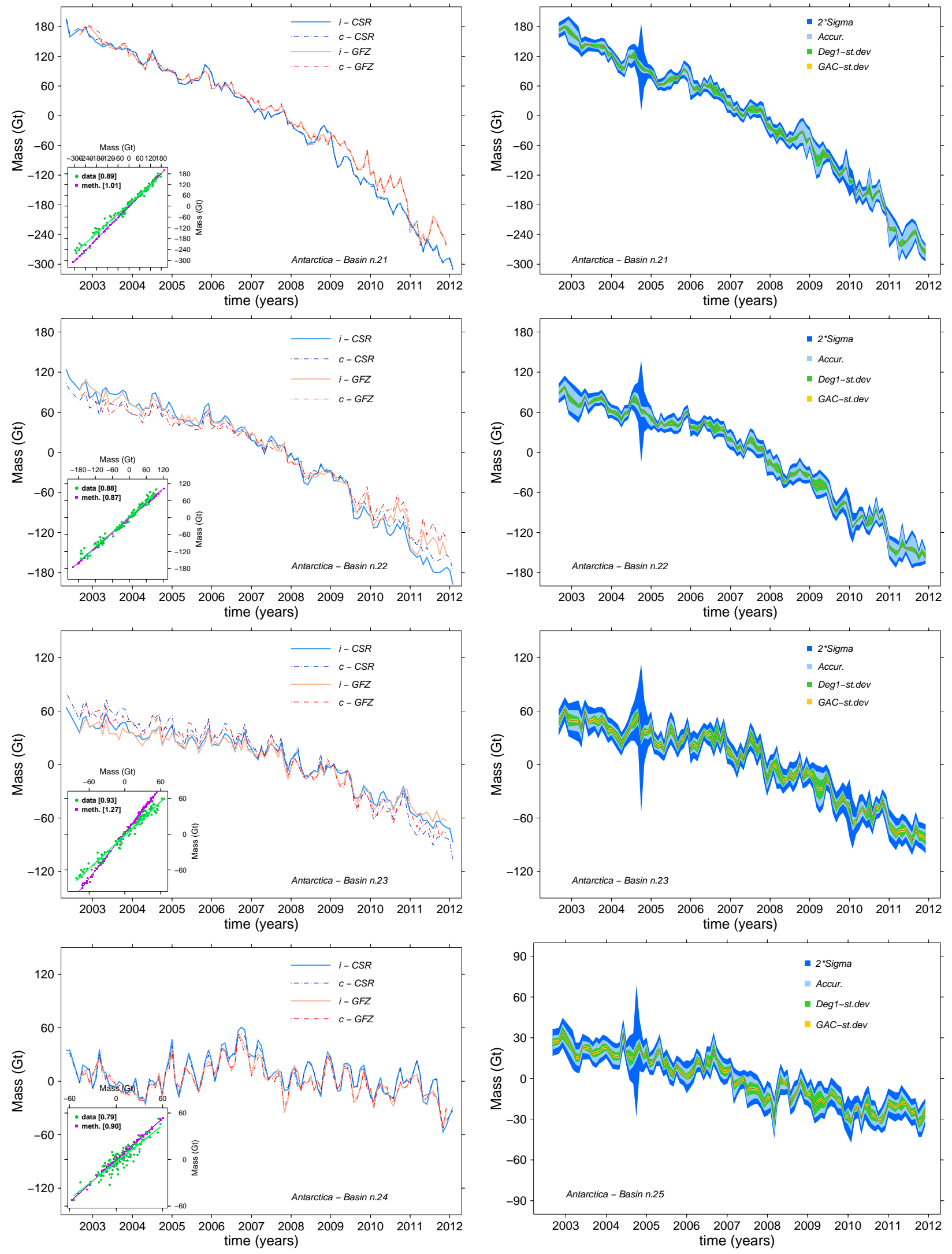

Fig. SM. 6. RL04 monthly solution for Antarctica. Basin number is indicated in each plot. Left column represents the comparison between the two methods and two data sets (CSR and GFZ) as in Fig.3 in the main text. Right column represents the monthly average solution where each color in the band around the average represents a contribution to the error estimate as in Fig. 7 of the main text. 

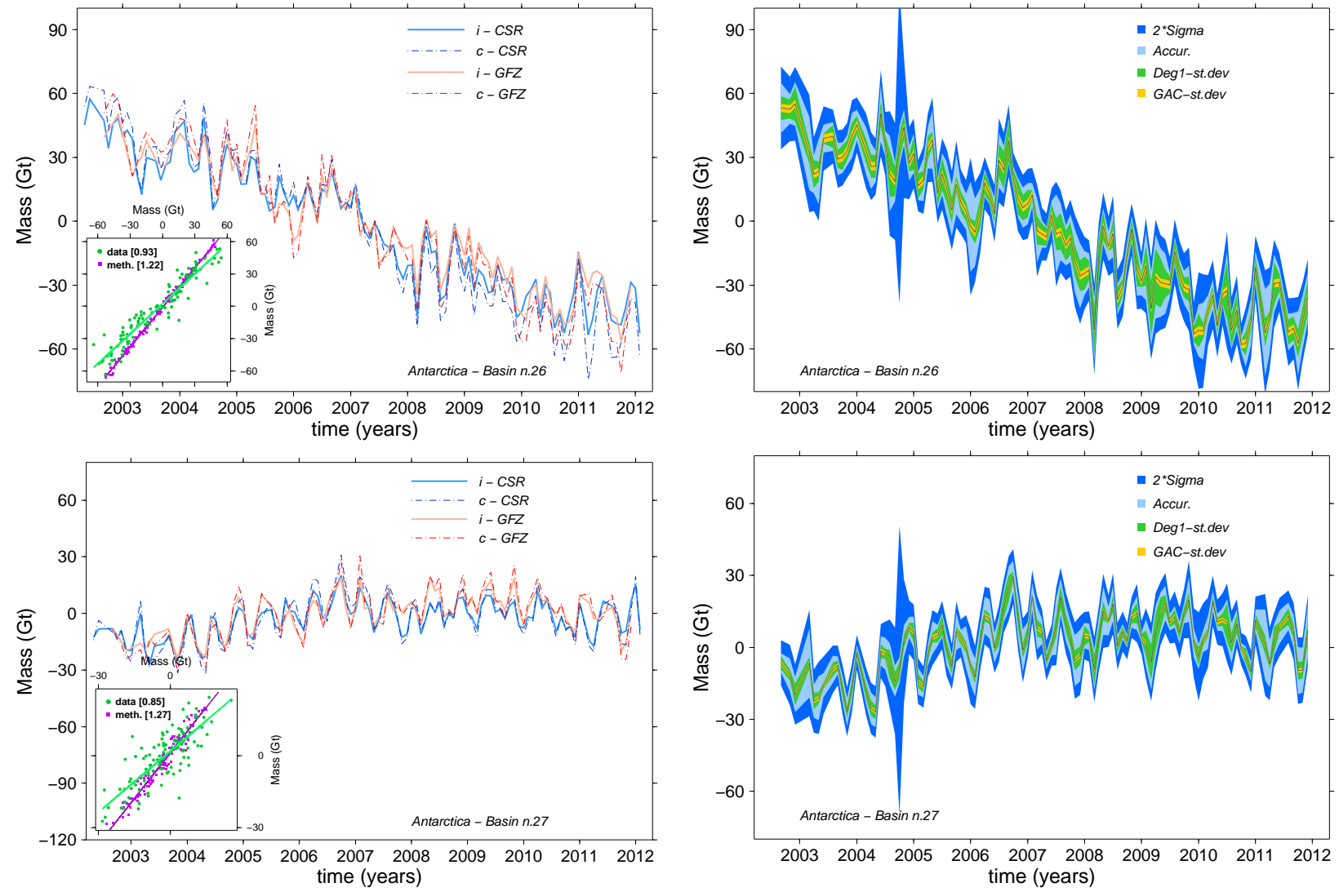

Fig. SM. 7. RL04 monthly solution for Antarctica. Basin number is indicated in each plot. Left column represents the comparison between the two methods and two data sets (CSR and GFZ) as in Fig.3 in the main text. Right column represents the monthly average solution where each color in the band around the average represents a contribution to the error estimate as in Fig. 7 of the main text. 

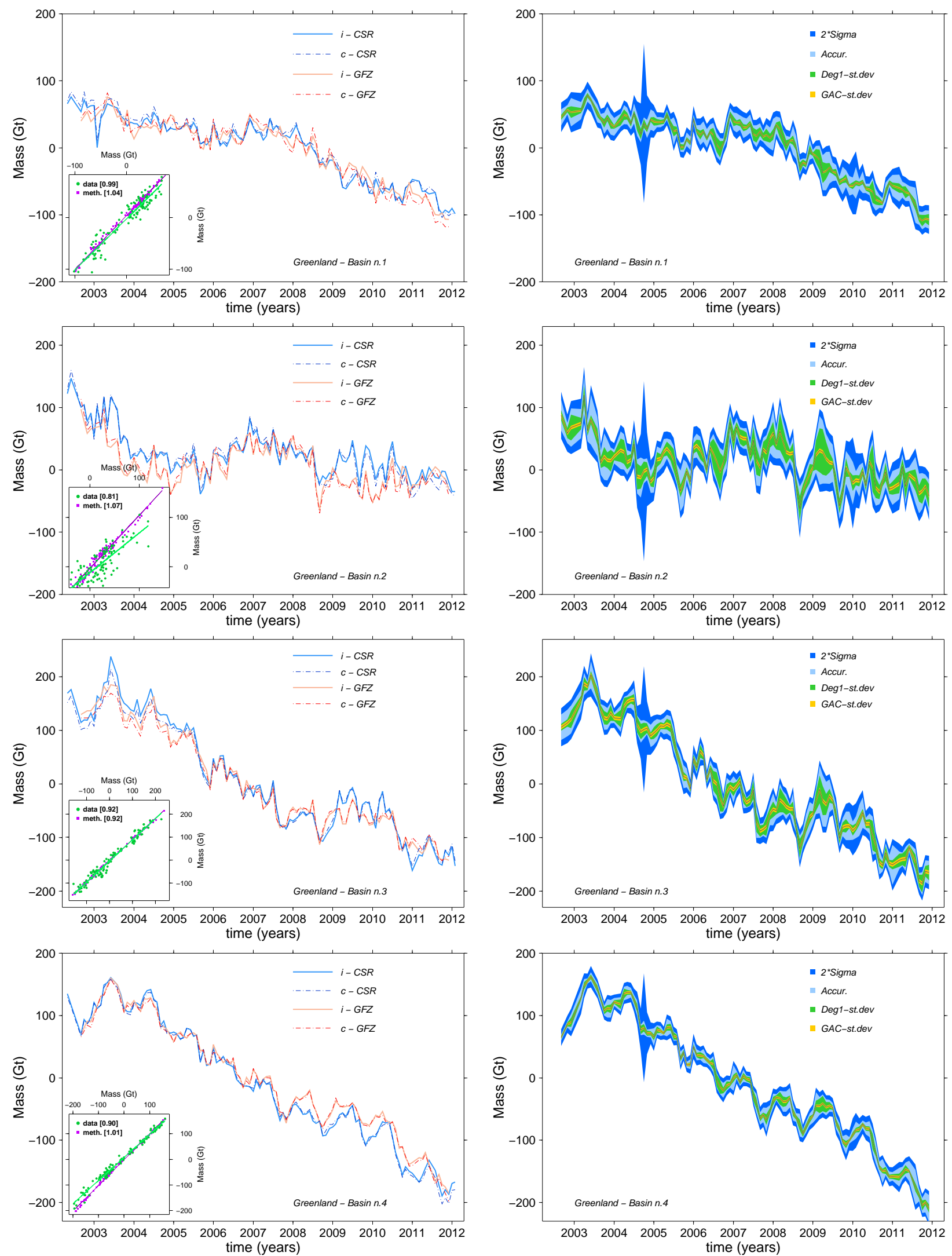

Fig. SM. 8. RL04 monthly solution for Greenland. Basin number is indicated in each plot. Left column represents the comparison between the two methods and two data sets (CSR and GFZ) as in Fig.3 in the main text. Right column represents the monthly average solution where each color in the band around the average represents a contribution to the error estimate as in Fig. 7 of the main text. 

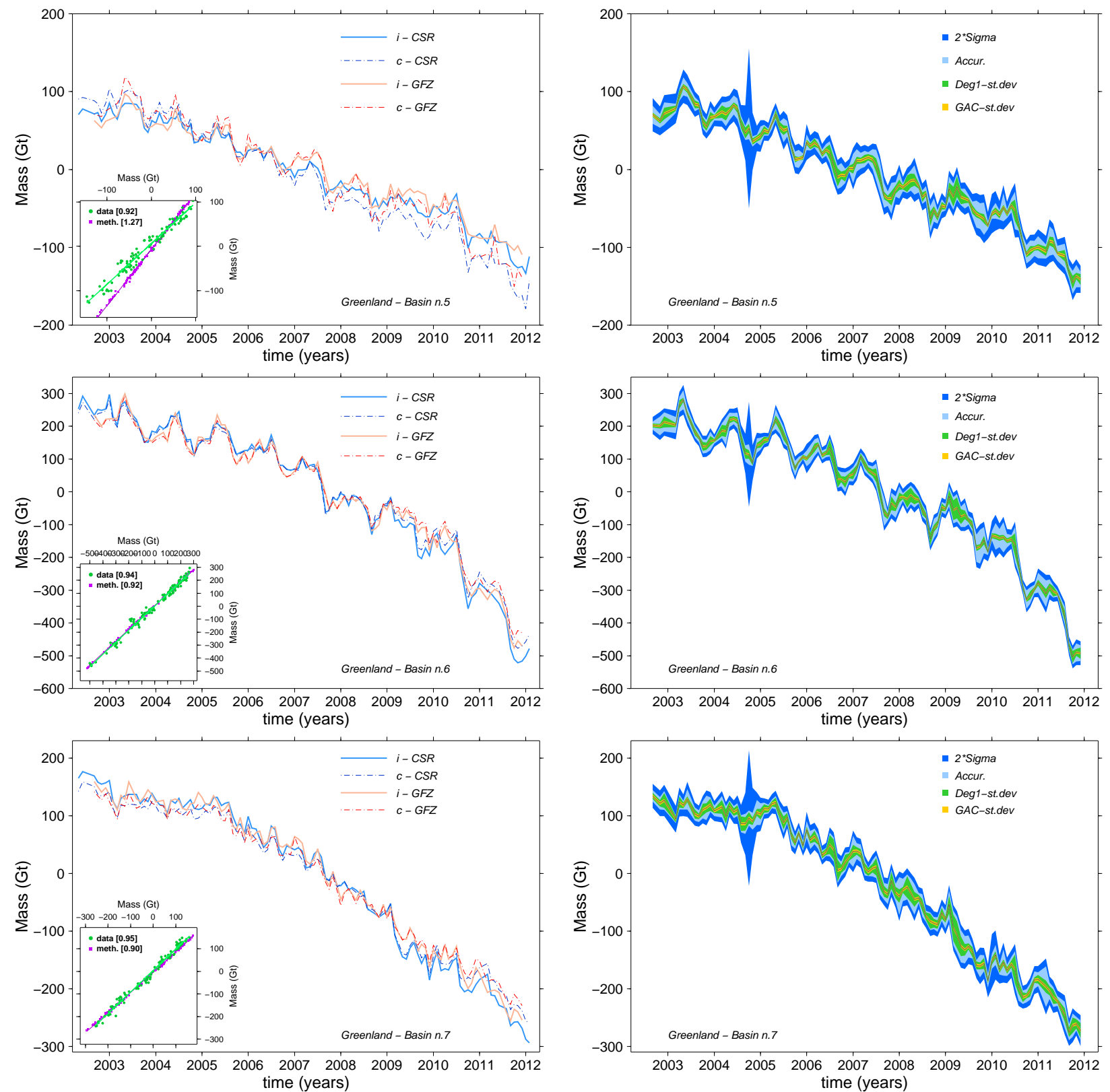

Fig. SM. 9. RL04 monthly solution for Greenland. Basin number is indicated in each plot. Left column represents the comparison between the two methods and two data sets (CSR and GFZ) as in Fig.3 in the main text. Right column represents the monthly average solution where each color in the band around the average represents a contribution to the error estimate as in Fig. 7 of the main text. 